\title{
Is there a subset of patients with recurrent cancer in the vagina who are not candidates for interstitial brachytherapy that can be treated with multichannel vaginal brachytherapy using graphic optimization?
}

\author{
Deepinder P. Singh, MD!, Kevin C. Bylund, MD!, Ahmad Matloubieh, MScl, Ali Mazloom, MD!, Alexander Gray, MSc', \\ Ravinder Sidhu, MD², Lucille Barrette, TA!' Yuhchyau Chen, MD, PhD'
}

'Department of Radiation Oncology, ${ }^{2}$ Department of Imaging Sciences, University of Rochester Medical Center, Rochester, NY, USA

\begin{abstract}
Purpose: To evaluate recurrent vaginal cancer treated with vaginal brachytherapy (VBT) using graphic optimization in patients not amenable to surgery and interstitial brachytherapy (ISBT).

Material and methods: We retrospectively reviewed the records of 5 patients with recurrent cancer in the vagina that were deemed not to be good candidates for ISBT implant because of medical reasons. All patients received computed tomography/magnetic resonance imaging (CT/MRI) based evaluation in addition to a detailed clinical examination, and were noted to have recurrent nodules in the vagina with size ranging from 10-25 mm. Four of the 5 patients had recurrent disease in the vaginal apex, whereas one patient had recurrence in the lateral vaginal wall. Subsequently, all patients were treated with external beam radiation therapy (EBRT) followed by multichannel vaginal cylinder (MVC)-based VBT using graphic optimization for shaping the isodose to improve the clinical target volume (CTV) coverage, as well as to spare the organs at risk (OAR). The dose to the bladder and rectum with regard to $0.1 \mathrm{cc}$, $1 \mathrm{cc}$, and $2 \mathrm{cc}$ were recorded.

Results: Median age of the patients was 78 years (range 58-86 years). Thickness of the lesions before VBT ranged from 6-15 mm. All patients were followed up with MRI at 3 months. All patients but one demonstrated complete clinical/ radiological response of the tumor. No patient had any grade III/IV toxicity at 24 months.

Conclusions: MVC-based VBT using graphic optimization is safe and yields favorable results if used judiciously.

J Contemp Brachytherapy 2015; 7, 2: 135-141 DOI: 10.5114/jcb.2015.51231
\end{abstract}

Key words: brachytherapy, graphic optimization, interstitial, vaginal cancer.

\section{Purpose}

Primary and recurrent vaginal cancer is extremely rare, and is one of the most challenging gynecological (GYN) cancers to treat because of its anatomic location with regard to adjacent critical structures [1,2]. Isolated vaginal recurrence from endometrial cancer constitutes about $70-75 \%$ of all recurrences and can be best managed either with surgery or radiation therapy (RT) [3-6]. Oncologic surgery may mandate extensive resection of tumor with wide surgical margins, but is not possible without sacrificing the adjacent rectum and bladder. Lack of organ sparing surgery makes RT the best treatment of choice [2]. With regard to external beam radiation therapy (EBRT), it is not possible to deliver very high tumoricidal doses even using intensity-modulated radiation therapy (IMRT) without affecting the rectum and bladder, although moderate doses of EBRT/IMRT can shrink the tumor in addition to sterilizing surrounding microscopic disease, while keeping the dose to critical organs with acceptable limits. Therefore, high doses of RT can be best delivered with a combination of EBRT followed by vaginal brachytherapy boost (VBT for $<5 \mathrm{~mm}$ thick lesions or ISBT implant for $>5 \mathrm{~mm}$ lesion thickness) [2,4,7-9]. Image guided brachytherapy (IGBT) has made it possible to deliver high doses more precisely and by sparing the critical organs [10-12].

Traditionally, we have used interstitial brachytherapy (ISBT) boost using ISBT template for lesions larger than 
$5 \mathrm{~mm}$ in thickness, but this technique requires anesthesia. Unfortunately, many uterine cancer patients in the U.S. have multiple co-morbidities that make them unfit for anesthesia, precluding an ISBT boost. Vaginal brachytherapy boost can also be delivered using a vaginal cylinder if the thickness of the lesion(s) is less than $5 \mathrm{~mm}$. A single-channel vaginal cylinder (SVC) is the most commonly used applicator for superficial lesions with more uniform dose distribution around the applicator that may not be ideal for thick and irregularly shaped lesions [1,2,13]. A multichannel vaginal cylinder (MVC) can offer better flexibility with regard to making dose distribution more conformal using optimization $[2,14,15]$. Some of the available optimization schemes, such as geometric and dose point optimization, fail to use the anatomic information [16]. Anatomy-based inverse optimization (IO) algorithm followed by graphic optimization can be used to decrease the incidence of toxicity [17]. Inverse optimization yields highly conformal dose coverage of the target volume and serves to spare critical organs that are near, or even inside, the target, and is therefore ideally suited for HDR brachytherapy [18]. The target coverage is compromised as a consequence of critical organ sparing, so graphic optimization was introduced in addition to IO where one can customize dose distribution manually by changing the isodose line by a mouse drag in any two-dimensional plane, which results in corresponding modifications of the dwell times and dwell positions [19].

We have been using SVC and MVC for a long period of time, but since the advent of IGBT with CT plus MRIbased planning, we started using high-dose rate (HDR) VBT using graphic optimization for patients who are not candidates for ISBT implant. The purpose of this retro- spective study was to look at the feasibility as well as acute and long-term tolerance/toxicity to the HDR treatment.

\section{Material and methods}

\section{Study population}

We retrospectively reviewed all patients with surgically managed endometrial adenocarcinoma with subsequent failure localized to the vagina. We found 5 patient records from 2010-2013. Patients had no history of previous EBRT and were not deemed to be candidates for surgery and ISBT due multiple medical co-morbidities. All patients received MRI of the pelvis with water-based vaginal gel and IV gadolinium evaluation using T2 weighted sequences in addition to the clinical examination, and were noted to have lesions sized between 10-25 mm (Table 1).

\section{Treatment}

All patients were planned for EBRT with the dose ranging from 45-50 Gy using IMRT to minimize dose to the rectum, bladder, and small bowel. Following completion of EBRT, all patients received further evaluation with a GYN exam and a repeat MRI at 3-4 weeks, allowing enough time for these larger pre-EBRT nodules to shrink. Unfortunately, all patients were noted to have residual disease between 6-15 $\mathrm{mm}$ in thickness (Table 1). All patients were then planned for IGBT using a Miami vaginal applicator (Elekta, Stockholm, Sweden) with $2.5 \mathrm{~mm}$ thin slice CT planning and MRI done after EBRT, which were fused to help with better delineation of the

Table 1. Age at presentation, stage, initial treatment, time to recurrence, initial size of the recurrent nodules before EBRT, EBRT details, size of the recurrent nodules before VBT, follow up in months, and disease status/remarks

\begin{tabular}{|c|c|c|c|c|c|c|c|c|c|}
\hline Age & $\begin{array}{c}\text { Stage } \\
\text { FIGO (TNM) }\end{array}$ & Initial Tx & $\begin{array}{l}\text { Time to } \\
\text { recurrence } \\
\text { (years) }\end{array}$ & $\begin{array}{l}\text { Tumor } \\
\text { size }(\mathrm{mm}) \\
\text { before } \\
\text { EBRT } \\
\end{array}$ & $\begin{array}{c}\text { EBRT } \\
\text { dose } \\
\text { Gy }\end{array}$ & $\begin{array}{l}\text { Tumor } \\
\text { size }(\mathrm{mm}) \\
\text { before } \\
\text { VBT } \\
\end{array}$ & $\begin{array}{l}\text { VBT dose } \\
\text { Gy/ } \\
\text { fractions }\end{array}$ & $\begin{array}{l}\text { RT to } \\
\text { last FU } \\
\text { (months) }\end{array}$ & $\begin{array}{l}\text { Disease status/ } \\
\text { remarks }\end{array}$ \\
\hline 86 & I A, Gr 1 (T1A, G1) & surgery & 3.5 & $15 \times 14$ & 54 & $8 \times 7$ & $5 \times 5$ & 36 & $\begin{array}{c}\text { NED loco-regionally/ } \\
\text { distant }\end{array}$ \\
\hline 81 & I A, Gr 3 (T1A, G3) & surgery & 20.0 & $25 \times 20$ & 54 & $12 \times 10$ & $5 \times 4$ & 32 & $\begin{array}{c}\text { NED loco-regionally/ } \\
\text { distant }\end{array}$ \\
\hline 64 & III, Gr 1 (T3, G1) & surgery & 1.0 & $16 \times 30$ & 45 & $15 \times 8$ & $5 \times 5$ & 24 & $\begin{array}{c}\text { NED locally } \\
\text { POD regionally/ } \\
\text { distant } \\
\text { DOD from liver } \\
\text { metastases }\end{array}$ \\
\hline 81 & I A, Gr 1 (T1A, G1) & surgery & 3.5 & $10 \times 8$ & 45 & $7 \times 6$ & $5 \times 2$ & 39 & $\begin{array}{c}\text { NED loco-regionally/ } \\
\text { distant } \\
\text { Diagnosed with low } \\
\text { grade bladder cancer }\end{array}$ \\
\hline 58 & II, Gr 2 (T2, G2) & $\begin{array}{l}\text { surgery + } \\
\text { VCBT }\end{array}$ & 1.5 & $10 \times 10$ & 50 & $8 \times 8$ & $5 \times 5$ & 12 & $\begin{array}{l}\text { Loco-regional } \\
\text { progression } \\
\text { DOD-lung \& brain } \\
\text { metastases }\end{array}$ \\
\hline
\end{tabular}


soft tissue extent of the disease (the Miami vaginal applicator contains 6 peripheral tubes, which are equally spaced around the surface of the cylinder for optimized treatment. The central tube/tandem was not used for any of our patients. The vaginal cylinder is $30 \mathrm{~mm}$ in diameter with cylinder sleeves of 35 and $40 \mathrm{~mm}$ ). We labeled all 6 peripheral tubes with numbers 1 through 6 in order to remember the orientation of the applicator and also for checking for any rotation. An immobilization device in the form of a board and clamp was used to secure the applicator in position (Fig. 1). The attending radiation oncologist contoured the target and critical organs. The clinical target volume (CTV) was defined based on a combination of clinical examination and pre- and post-EBRT MRI findings. The length and circumference for CTV was based on pretreatment extent of disease, whereas thickness was based on post-EBRT MRI and clinical examination [2]. The CT scans were used for treatment planning, which were performed with the Oncentra Brachytherapy Planning System (Elekta, Stockholm, Sweden). For each session of HDR, individualized CT/MR planning was done using graphic optimization for shaping the isodose to improve the CTV coverage as well as to spare the organs at risk (OAR). The dose to the bladder and rectum with regards to $0.1 \mathrm{cc}, 1 \mathrm{cc}$, and $2 \mathrm{cc}$ were recorded. The plan was optimized to cover at least $90 \%$ of $C T V\left(D_{90}\right)$ with prescription dose, and to minimize dose to the critical organs including bladder, rectum, sigmoid, and urethra where applicable. The 2 cc rectal dose was kept $<75$ Gy and bladder dose $<90$ Gy. All patients received HDR brachytherapy to 1025 Gy in 2-5 fractions, 1-2 times per week.

\section{Follow-up}

Follow-up ranged from 12-36 months (median 24 months). All patients were scheduled for follow-up and a GYN examination initially at 4 weeks for monitoring the early side effects of RT, then at 3 month intervals for the first 2 years, and then every 6 months thereafter. All patients received a repeat MRI at 12 weeks for post-treatment evaluation to determine outcome. The response was defined by RECIST criteria (measured in at least one dimension; longest diameter in the plane of measurement to be recorded) [20]. Toxicity was reported according to CTCAE v4.

\section{Results}

Median age of the patients was 78 years (range $58-86$ years). Details regarding the initial diagnosis and stage are described in Table 1. Four of the five patients had recurrent disease in the vaginal apex and one patient had disease in the right lateral vaginal wall (Figs. 2, 3). The length of the vagina involved the upper one-third in four patients, and mid to lower vagina in the fifth patient. Four of the five patients showed near total complete local response seen on the follow-up MRI (Fig. 4), whereas one patient showed partial local response as well as progression of disease in the lungs and brain. The $D_{90}$ was $90 \%$ in most of patients with BED ranging from 74.5 to $110.2 \mathrm{~Gy}$ (median BED 90.6 Gy) and EQD2 ranging from 62.1 to

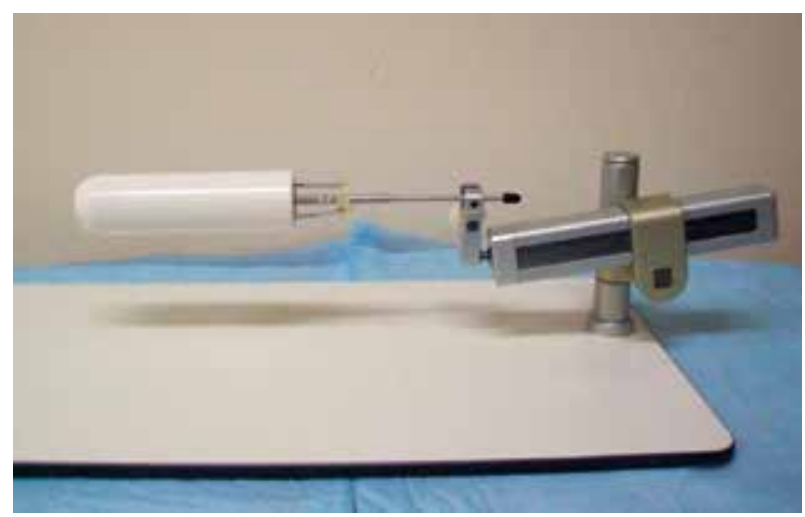

Fig. 1. Immobilization device used for securing applicator in position during planning and treatment

91.8 Gy (median EQD2 75.6 Gy). One patient developed hematuria following EBRT, and upon cystoscopy was noted to have a superficial bladder tumor that was confirmed on biopsy and managed surgically. Regarding local side effects, one patient was noted to have narrowing of the vagina as well as some telangiectasia, but no mucosal ulceration was ever noted during her 36 months follow-up. Three patients showed initial grade I/II vaginal radiation mucositis that subsided in 4-6 weeks' time with conservative management without long term sequelae; one of these three patients developed regional as well as distant failure and died of metastatic liver disease at 24 months. No patient was noted to have any long-term grade III or IV toxicity from the combined treatment. The fifth patient showed no response to the treatment initially, but then demonstrated disease progression, both loco-regionally as well as distant metastases (lung and brain) that were treated with palliative XRT. She eventually succumbed to her disease at 12 months.

\section{Discussion}

Brachytherapy plays a vital role in the management of recurrent endometrial cancer in the vagina, especially isolated recurrences that are not amenable to surgery $[2,21,22]$. A combination of EBRT and VBT (VBT for $<5 \mathrm{~mm}$ thick lesions or ISBT implant for $>5 \mathrm{~mm}$ lesion thickness) is best suited to deliver a total dose of $80-85$ Gy that is required to control the disease $[1,2,6,23]$. This combination can salvage up to $40-100 \%$ of patients in previously non-irradiated cases, whereas the salvage rates may be as low as $10-25 \%$ in previously treated patients $[4,6,23,24]$. External beam radiation therapy using IMRT followed by ISBT boost is done more often in such situations and less commonly a combination of EBRT and SVC. There are situations, however, when we come across patients with recurrent vaginal lesions that are big enough to preclude VBT using SVC, and also are not amenable to ISBT due to not being a candidate for anesthesia. The MVC applicator delivers a unique solution by providing increased dose conformity akin to interstitial implants through the placement of a simple cylinder in the outpatient setting [2]. Some studies have shown that HDR brachytherapy salvage for vaginal recurrences using SVC has a high likelihood of late toxicity as well as local morbidity from high 

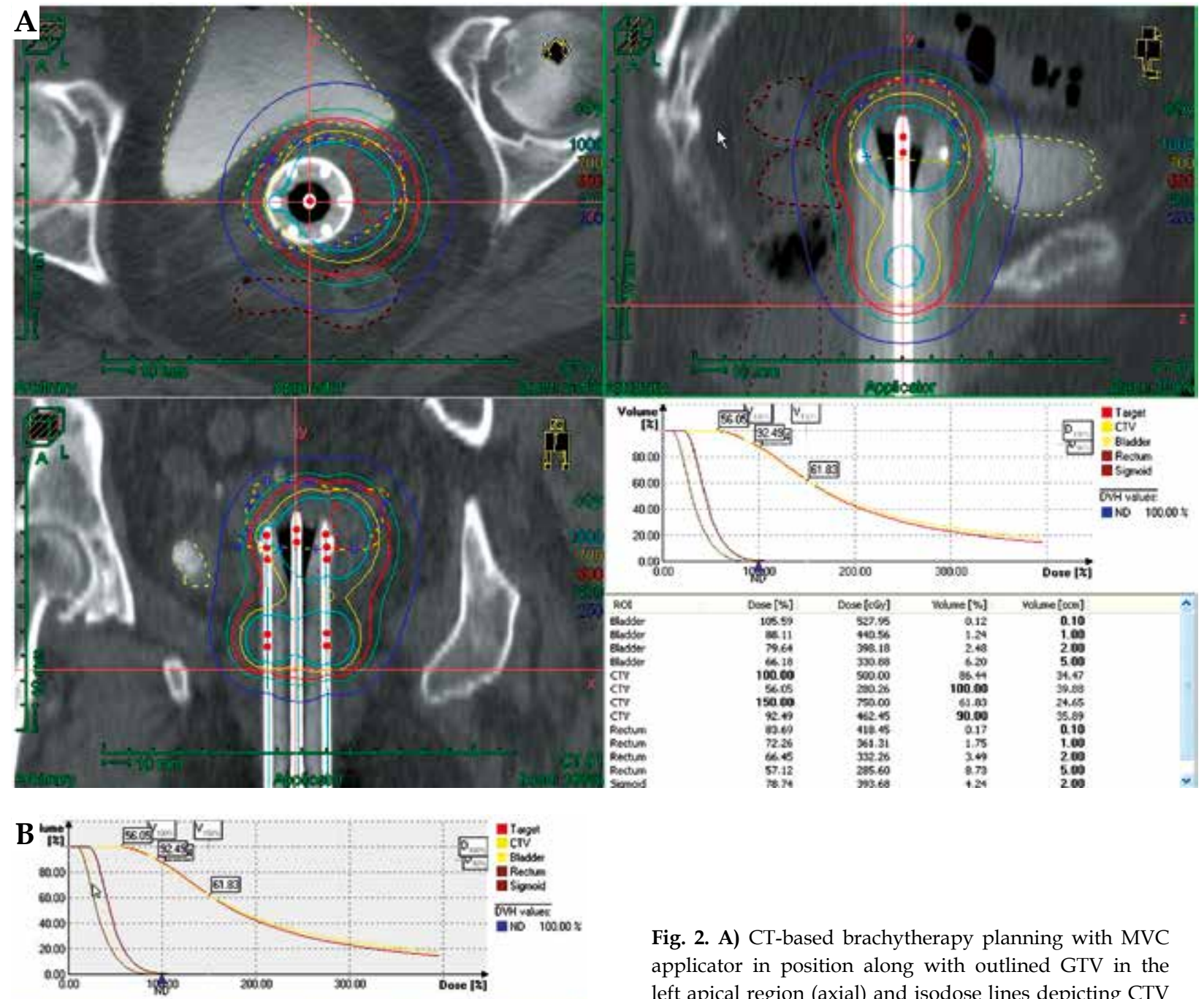

\begin{tabular}{|c|c|c|c|c|c|}
\hline 200 & $\operatorname{Dese}[\%]$ & 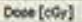 & Nolene[s] & Votune $[00$ is] & A \\
\hline Elesder & 106.5 & 52.95 & 0.12 & 0.10 & \\
\hline Exdore & $\$ .11$ & +00.56 & 1.24 & 1.00 & \\
\hline Elodder & met & 300.10 & 201 & 200 & \\
\hline Hodder & 4.18 & 300.6 & 620 & 500 & \\
\hline $\mathrm{CN}$ & 10000 & 800.00 & 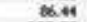 & 9.45 & \\
\hline $\mathrm{cNV}$ & 308 & 200.6 & 160.00 & nos & \\
\hline $\mathrm{ctv}$ & 150.00 & 700.00 & 61.83 & 24.65 & \\
\hline $\mathrm{CV}$ & 22.49 & $\$ 2.45$ & 30.00 & 3.89 & \\
\hline Bectum & 83.69 & 418.45 & 0.17 & 010 & \\
\hline Bache & $n 2.26$ & $\begin{array}{l}x_{1} 1, j n \\
m, x\end{array}$ & 1.78 & 100 & \\
\hline Rentum & 66.45 & 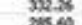 & 247 & $\begin{array}{l}200 \\
500\end{array}$ & \\
\hline Seromit & $\begin{array}{l}59.12 \\
72.74\end{array}$ & 25.60 & 80 & 200 & $\downarrow$ \\
\hline
\end{tabular}

Fig. 2. A) CT-based brachytherapy planning with MVC applicator in position along with outlined GTV in the left apical region (axial) and isodose lines depicting CTV coverage as well as dose to the adjacent rectum/bladder. Clockwise: axial, sagital, DVH, and coronal planes. B) The DVH graph (enlarged from Fig. 2A) shows percent of volume (target, CTV, bladder, rectum, sigmoid) vs. percent of dose being received. The table below the DVH shows dose in percent, and cGy for percent of volume and $\mathrm{ccm}$ volume of target, CTV, bladder, rectum, sigmoid

dose to vaginal mucosa $[2,23,24]$, whereas modern IGBT using MVC is expected to reduce these morbidities and late toxicities [2]. Tumors involving lateral vaginal walls treated with VBT are likely to have less toxicity as dose to the critical organs and uninvolved vagina can be better restricted compared to the vaginal apex lesions or involving anterior/post vaginal wall abutting the rectum and bladder [2]. The geometric and dose point optimizations are based only on the location of the active dwells, since these methods necessarily result in an approximation of the shape of the anatomy and reduce the clinical target volume to a geometric representation without regard to anatomic relationships, resulting in an overdosage of normal tissue $[16,18]$. With efforts to reduce the dose to normal organs at risk of radiation injury as well for complete coverage of the tumor, the dose distribution should be as conformal as possible to the relevant anatomy. An anatomy-based dose optimization algorithm has been developed to automatically and rapidly produce conformal dose coverage of the target volumes while minimizing the dose to the organs at risk in the delivery of HDR brachytherapy. The dwell times are optimized using a simulated annealing algorithm governed entirely by the anatomy extracted from a CT and by prescribed dose constraints on each anatomic volume. This inverse planning simulated annealing (IPSA) algorithm has been used for several anatomic sites (gynecologic, prostate etc.) [16].

A study by Akimoto et al. comparing anatomy-based inverse optimization with geometric optimization showed that percentage of volume receiving $80-150 \%$ of the prescribed minimal peripheral dose $\left(\mathrm{V}_{80}-\mathrm{V}_{150}\right)$ of the 


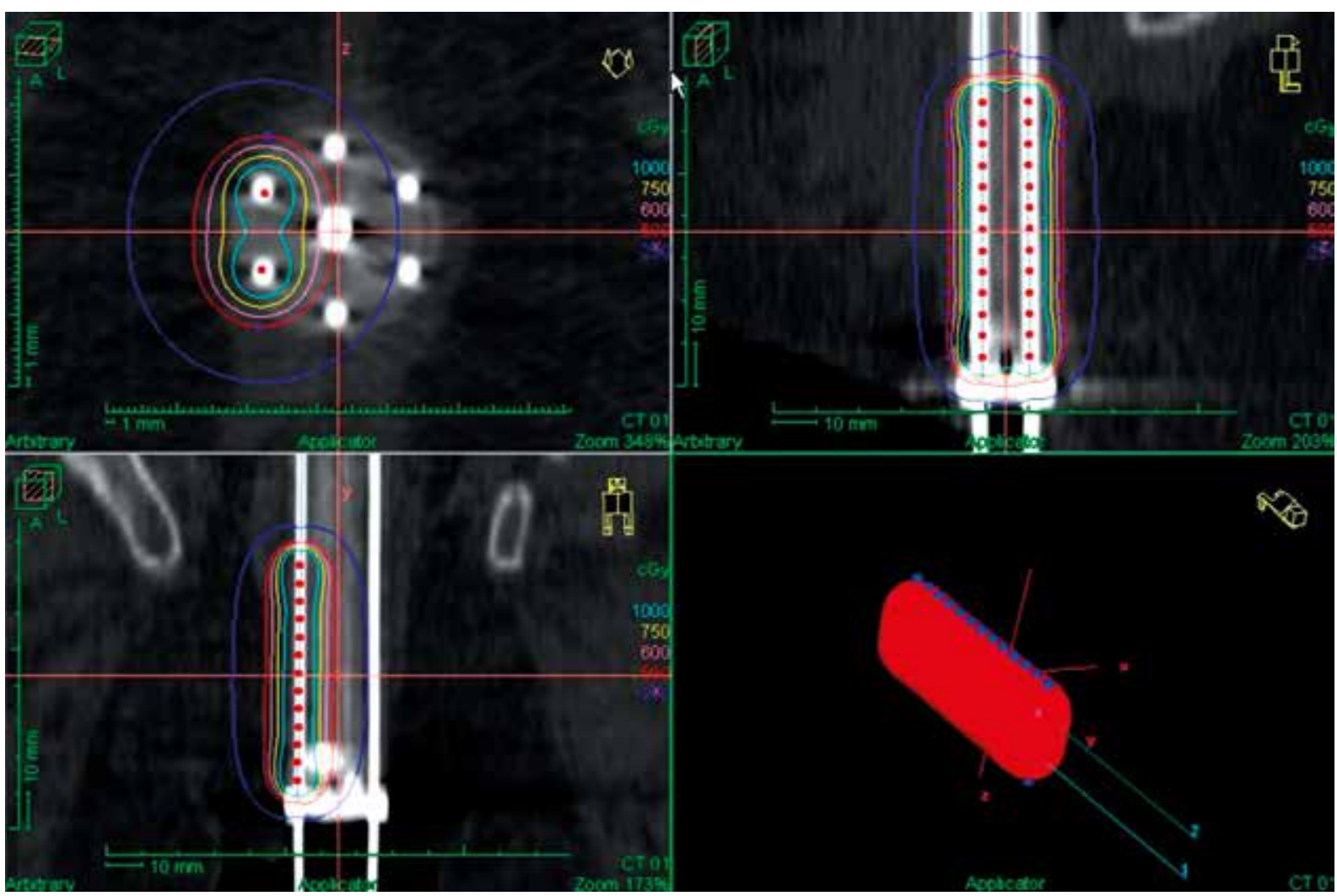

Fig. 3. CT-based brachytherapy planning with MVC applicator in position (right lateral vaginal wall) along with isodose lines depicting CTV coverage. Clockwise: axial, sagital, coronal, and 3D planes

critical organ (urethra) generated by anatomy-based IO were significantly lower than the corresponding values generated by $\mathrm{GO}$, as well as minimal dose received by $5-50 \%$ of the target volume $\left(D_{5}-D_{50}\right)$ obtained using anatomy-based IO were significantly lower than those obtained using GO. They showed that acute toxicity was significantly lower in patients for whom anatomy-based IO $(16 \%)$ was used than in those for whom GO was used $(37 \%)$, consistent with the reduced critical organ (urethra) dose $(p<0.01)$. Studies have shown that IO followed by graphic optimization yields superior target coverage [19]. Considering the difference in the dose non-uniformity ratio and homogeneity index between geometric optimization and anatomy-based IO, the latter method with graphic optimization yielded significantly improved dose homogeneity and a significant decrease in the volume of hot spots compared with that generated by geometric optimization anatomy-based IO followed by graphic optimization for planning HDR brachytherapy for prostate cancer yielded reduced urethral doses compared with standard GO, leading to a reduction in the incidence of Grade 2 or worse acute GU toxicity. The results of this study suggest that anatomy-based IO is superior to GO; hence, routine application of anatomy-based IO followed by graphic optimization is recommended [19]. We were able to use geometric optimization anatomy-based IO followed by graphic optimization for planning HDR brachytherapy.

Our aim was to deliver a dose of 80-85 Gy from a combination of EBRT and brachytherapy $[1,2,6,23]$, but know- ing that these patients weren't candidates for ISBT under anesthesia due to medical problems, we initially prescribed a dose of 50-54 Gy with EBRT with the thought that a larger dose may be able to shrink these recurrent nodules to $<5 \mathrm{~mm}$, hence making them suitable for VBT using standard SVC. After the first few patients, we tried to bring down the EBRT dose subsequently between 45-50 Gy. In our patients, the BED ranged from 74.5 to 110.2 Gy (median BED 90.6 Gy), and EQD2 ranged from 62.1 to $91.8 \mathrm{~Gy}$ (median EQD2 75.6 Gy). Unfortunately, one of our patients developed low grade bladder cancer during the course of her treatment, and her final radiation dose needed to be truncated that resulted in this wide range for our BED and EQD2 dose.

One must exercise caution while prescribing brachytherapy dose to thick tumors using MVC, as the dose to the vaginal surface could be more than $150 \%$ of the dose prescribed to depth. This raises the possibility of high local morbidity as well as toxicity to adjacent critical structures. At our institute, we treated patients with a tumor thickness of 6-15 mm and achieved excellent local control without any significant local morbidities or late toxicities. $\mathrm{D}_{90}$ was $90 \%$ in most of our patients with less than $60 \%$ of the CTV receiving dose $\leq$ to $150 \%$ and only about $20 \%$ of the CTV receiving dose around $300 \%$ (Fig. 2). The thickness of the lesion that can be safely treated with VBT using MVC is being reported from some institutions now. A recently published study from a larger U.S. gynecological center recommended limiting this approach using MVC to a tumor thickness of $7 \mathrm{~mm}$ or 


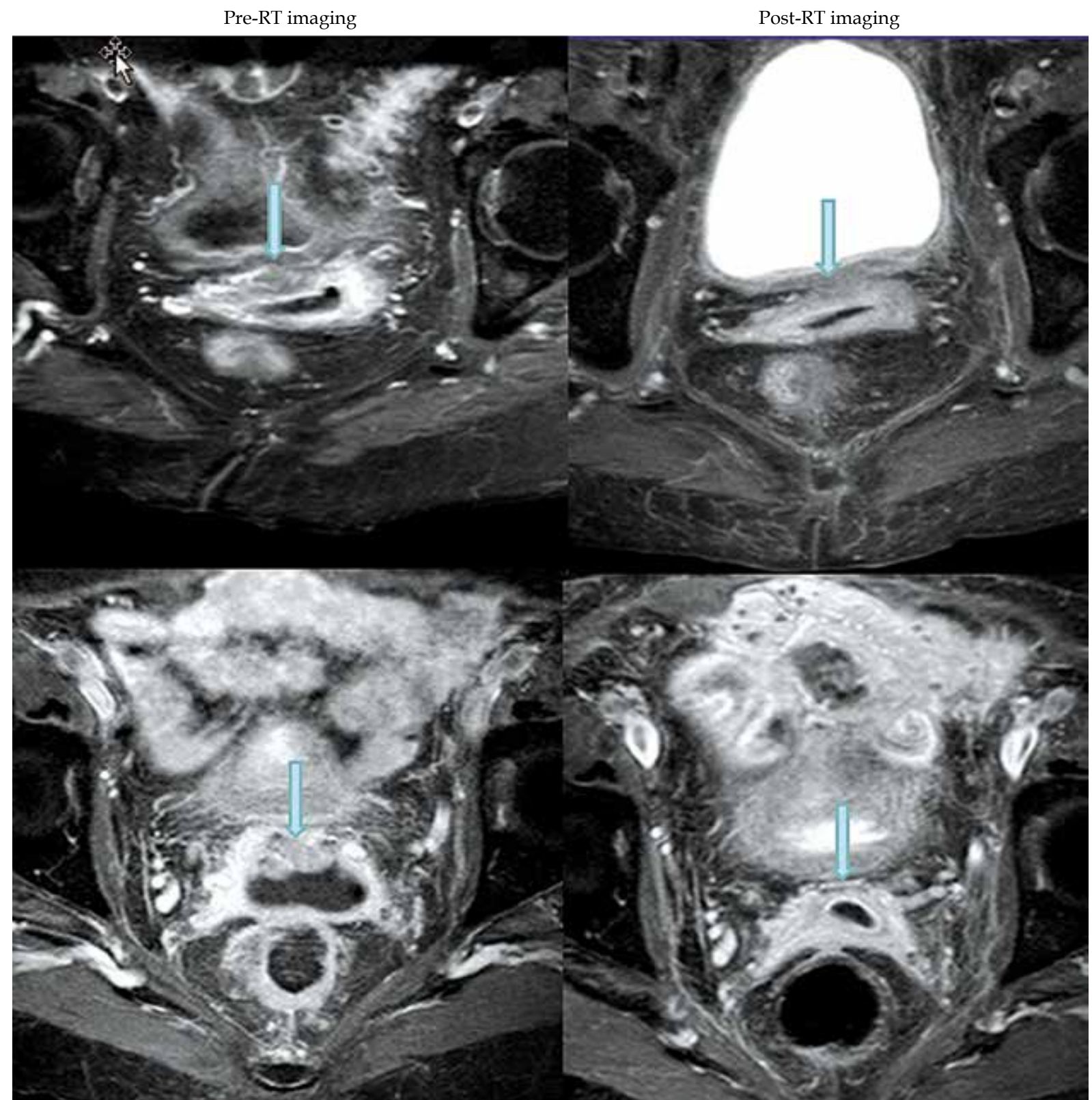

Fig. 4. MRI for Case \#1 (upper row) and Case \#3 (lower row) with arrow showing the pretreatment apical nodule on the left side and corresponding post-treatment response on the right

less at the time of brachytherapy [2]. We are currently hoping to treat more patients using this technique, as well as working on doing long-term follow-up on these patients treated in the past. More experience is needed to define the dose per fraction and thickness of the tumor that can be safely treated. MVC requires a more complex planning process in addition to customized IGBT planning for each fraction, and must be done by experienced physicists and brachytherapists [2].

\section{Conclusions}

Multichannel vaginal cylinder brachytherapy using graphic optimization is feasible in a select group of pa- tients who are not candidates for ISBT, but extreme care should be taken in keeping the dose to OAR below tolerance.

\section{Acknowledgments}

The authors wish to thank Mrs. Laura Finger for editorial assistance.

Presented at the American Brachytherapy Society's 35th Annual Meeting (April 3-5, 2014) in San Diego, CA, USA.

\section{Disclosure}

Authors report no conflict of interest. 


\section{References}

1. Lian J, Dundas G, Carlone M et al. Twenty year review of radiotherapy for vaginal cancer: an institutional experience. Gynecol Oncol 2008; 111: 298-306.

2. Kim H, Rajagopalan MS, Houser C, Beriwal S. Dosimetric comparison of multichannel with one single-channel vaginal cylinder for vaginal cancer treatments with high-dose-rate brachytherapy. Brachytherapy 2014; 13: 263-267.

3. Keys HM, Roberts JA, Brunetto VL et al. A phase III trial of surgery with or without adjunctive external pelvic radiation therapy in intermediate risk endometrial adenocarcinoma: a Gynecologic Oncology Group study. Gynecol Oncol 2004; 92: 744-751.

4. Creutzberg CL, van Putten WL, Koper PC et al. Survival after relapse in patients with endometrial cancer: results from a randomized trial. Gynecol Oncol 2003; 89: 201-209.

5. Nout RA, van de Poll-Franse LV, Lybeert ML et al. Longterm outcome and quality of life of patients with endometrial carcinoma treated with or without pelvic radiotherapy in the postoperative radiation therapy in endometrial carcinoma 1 (PORTEC-1) trial. J Clin Oncol 2011; 29: 1692-1700.

6. Vargo JA, Kim H, Houser CJ et al. Definitive salvage for vaginal recurrence of endometrial cancer: the impact of modern intensity-modulated-radiotherapy with image-based HDR brachytherapy and the interplay of the PORTEC 1 risk stratification. Radiother Oncol 2014; 113: 126-131.

7. Stryker JA. Radiotherapy for vaginal carcinoma: a 23-year review. Br J Radiol 2000; 73: 1200-1205.

8. Samant R, Tam T, Dahrouge S, E C. Radiotherapy for the treatment of primary vaginal cancer. Radiother Oncol 2005; 77: 133-136.

9. Frank SJ, Jhingran A, Levenback C, Eifel PJ. Definitive radiation therapy for squamous cell carcinoma of the vagina. Int J Radiat Oncol Biol Phys 2005; 62: 138-147.

10. Dimopoulos JC, Pötter R, Lang $S$ et al. Dose-effect relationship for local control of cervical cancer by magnetic resonance image-guided brachytherapy. Radiother Oncol 2009; 93 : 311-315.

11. Beriwal S, Heron DE, Mogus R, et al. High dose rate brachytherapy (HDRB) for primary or recurrent cancer in the vagina. Radiat Oncol 2008; 3: 7.

12. Beriwal S, Bhatnagar A, Heron DE et al. High-dose-rate interstitial brachytherapy for gynecologic malignancies. Brachytherapy 2006; 5: 218-222

13. Hasbini A, Haie-Meder C, Morice P et al. Outcome after salvage radiotherapy (brachytherapy $+/$ - external) in patients with a vaginal recurrence from endometrial carcinomas. Radiother Oncol 2002; 65: 23-28.

14. Syh JH, Wu T, Syh J et al. An optimization study and comparison of a multichannel vaginal cylinder versus single channel cylinder applicator in high dose rate brachytherapy. Int J Radiat Oncol Biol Phys 2012; 84: S456-S457.

15. Peng J, Sinha R, Patel R et al. A comparison of 3D dose planning regimens for vaginal cuff brachytherapy using a flexible inflatable multi- channel gynecologic applicator. Brachytherapy 2011; 10 (Suppl 1): S67.

16. Lessard E, Hsu IC, Pouliot J. Inverse planning for interstitial gynecologic template brachytherapy: Truly anatomy-based planning. Int J Radiat Oncol Biol Phys 2002; 54: 1243-1251.

17. Kolkman-Deurloo IK, Deleye XG, Jansen PP, Koper PC. Anatomy based inverse planning in HDR prostate brachytherapy. Radiother Oncol 2004; 73: 73-77.

18. Hsu IC, Lessard E, Weinberg V, Pouliot J. Comparison of inverse planning simulated annealing and geometrical optimization for prostate high-dose-rate brachytherapy. Brachytherapy 2004; 3: 147-152.
19. Akimoto T, Katoh H, Kitamoto $Y$ et al. Anatomy based inverse optimization in high dose rate brachytherapy combined with hypofractionated external beam radiotherapy for localized prostate cancer: Comparison of incidence of acute genitourinary toxicity between anatomy-based inverse optimization and geometric optimization. Int J Radiat Oncol Biol Phys 2006; 64: 1360-1366.

20. Eisenhauer EA, Therasse P, Bogaerts J et al. New response evaluation criteria in solid tumors: Revised RECIST guidelines version (1.1). Eur J Cancer 2009; 45: 228-247.

21. Mock U, Kucera H, Fellner C et al. High-dose rate (HDR) brachytherapy with or without external beam radiotherapy in the treatment of primary vaginal carcinoma: Long-term results and side effects. Int J Radiat Oncol Biol Phys 2003; 56: 950-957.

22. Perez CA, Grigsby PW, Garipagaoglu M et al. Factors affecting long-term outcome of irradiation in carcinoma of the vagina. Int J Radiat Oncol Biol Phys 1999; 44: 37-45.

23. Petignat P, Jolicoeur M, Alobaid A et al. Salvage treatment with high-dose-rate brachytherapy for isolated vaginal endometrial cancer recurrence. Gynecol Oncol 2006; 101: 445-449.

24. Jhingran A, Burke TW, Eifel PJ. Definitive radiotherapy for patients with isolated vaginal recurrence of endometrial carcinoma after hysterectomy. Int J Radiat Oncol Biol Phys 2003; 56: 1366-1372. 